\title{
AVALIAÇÃO NUTRICIONAL E RISCO DE DESNUTRIÇÃO EM IDOSOS COM DEMÊNCIAS
}

\section{Annah Rachel Graciano}

Discente do curso de medicina do Centro Universitário de Anápolis, Goiás (GO), Brasil.

\section{Andressa Meline Cozer}

Discente do curso de medicina do Centro Universitário de Anápolis, Goiás (GO), Brasil.

\section{Vítor Marcilio Lima Santana}

Discente do curso de medicina do Centro Universitário de Anápolis, Goiás (GO), Brasil.

\section{Júlia Maria Rodrigues de Oliveira}

Mestre em Ciências da saúde; Discente do Centro Universitário de Anápolis, Goiás (GO), Brasil.

\begin{abstract}
RESUMO: Aplicar o MNA como instrumento de triagem nutricional em idosos com a finalidade de identificar os desafios e sua aplicabilidade na rotina da nutrição desses pacientes. Coorte prospectiva, realizada entre janeiro de 2014 a junho de 2017, baseada em 300 idosos entre 60 e 95 anos, que foram submetidos a avaliações geriátricas específicas. Determinou-se a frequência de desnutrição e o risco de desnutrição em grupos com demências comparadas a idosos sem demência ou transtorno cognitivo leve. O estado nutricional normal prevaleceu em idosos sem demência $(58,4 \%)$. A prevalência entre idosos com demência foi de 18,2\% a 33,3\%. Idosos com Parkinson estiveram mais relacionados à desnutrição (33,3\%) que idosos com outras demências. Os pacientes com doença de Alzheimer foram os que apresentaram maior risco de desnutrição (63,6\%). Há relação positiva entre o desenvolvimento de desnutrição e demências, principalmente associada à doença de Parkinson.
\end{abstract}

PALAVRAS-CHAVE: Desnutrição; Doença de Alzheimer; Avaliação nutricional.

\section{NUTRITIONAL ASSESSMENT AND MALNUTRITION RISKS IN ELDERLY PEOPLE WITH DEMENTIA}

\begin{abstract}
Current study comprised the application of MNA as a nutrition selection test in elderly people to identify challenges and applicability in the patients' nutrition routine. A cohort perspective was undertaken, between January 2014 and June 2017, with 300 elderly people, aged 60 - 95 years, submitted to specific geriatric evaluations. Malnutrition frequency and malnutrition risks in groups with dementia was determined and compared to others without dementia or with slight cognitive disorder. Normal nutritional status was predominant in elderly people without signs of dementia (58.4\%), whilst the prevalence among elderly people with dementia ranged between 18.2\% and 33.3\%. Elderly people with Parkinson's disease were related to malnutrition (33.3\%) rather than elderly with other types of dementia. Patients with Alzheimer's disease had the highest malnutrition risk (63.6\%). There is a positive relationship between the development of malnutrition and dementia, mainly associated with Parkinson's disease.
\end{abstract}

KEY WORDS: Malnutrition; Alzheimer Disease; Nutrition Assessment. 


\section{INTRODUÇão}

A Organização Mundial de Saúde (OMS) define o idoso como aquele indivíduo com 60 anos de idade ou mais, limite este válido apenas para os países em desenvolvimento, visto que nos países desenvolvidos admite-se um nível de corte de 65 anos de idade. Já a Política Nacional do Idoso (PNI), lei $\mathrm{n}^{0} 8.842$, de 4 de janeiro de 1994, e o estatuto do Idoso, lei $\mathrm{n}^{\mathrm{o}} 10.741$, de $1^{\mathrm{o}}$ de outubro de 2003 , definem idoso pessoas com 60 anos ou mais ${ }^{1}$.

Projeções estatísticas da OMS, para 2025, apontam que a população brasileira quintuplicará, enquanto que o grupo etário com idade superior a 60 anos será 15 vezes maior, representando 34 milhões de pessoas. Sendo assim, o Brasil ocupará o sexto lugar do mundo em contingente de idosos. O envelhecimento caracteriza-se por um processo natural e individual, que ocasiona uma série de mudanças fisiológicas, metabólicas, anatômicas, sociais e psicológicas, que se manifestam em mudanças estruturais e funcionais ${ }^{1}$.

O envelhecimento, um acontecimento normal dentre os organismos vivos, ocorre de forma gradual, resultando na diminuição global da capacidade funcional do indivíduo. Estas modificações são influenciadas por diversas variáveis, como as biológicas, sociodemograficas e sociais, o que cursa com algumas limitações no idoso, tornando este indivíduo mais susceptível a diversas enfermidades, fator que diminui a sua qualidade de vida ${ }^{2}$.

$\mathrm{O}$ aumento da esperança média de vida não é sinônimo de melhor qualidade de vida, e a nutrição é um fator que contribui de forma positiva para melhoria da saúde destes indivíduos. Entre os vários fatores que influenciam na qualidade de vida do idoso, estão aqueles que afetam a ingestão alimentar e estes são vistos como fatores facilitadores para a ocorrência da má nutrição no idoso $^{3}$.

A avaliação nutricional do idoso é parte integrante da Avaliação Geriátrica Ampla (AGA) por ser uma ferramenta sensível de detecção dos fatores de risco associados à desnutrição. Assim, torna-se relevante o desenvolvimento de métodos que utilizem questões simples e rápidas que permitam destacar sinais de alerta do estado nutricional e direcionem as intervenções que devem ser realizadas pelos profissionais da área da saúdé⿱ .
Há diversas ferramentas disponíveis e validadas, na literatura, para triagem nutricional. É comum a comparação entre essas ferramentas, mas é necessário estabelecer senso crítico sobre a mais indicada ao ambiente em que será realizada. Não há consenso sobre o melhor instrumento, pois os descritos na literatura possuem limitações, vantagens e desvantagens quando utilizados em populações específicas. Não há padrão para a triagem nutricional no Brasil. Portanto, a escolha da ferramenta mais adequada deve considerar os objetivos do rastreamento nutricional, os recursos disponíveis, assim como a população atendida ${ }^{5}$.

De acordo com esse mesmo autor, a MiniAvaliação Nutricional (MNA) consiste de questões e medidas antropométricas para determinar um escore indicador de desnutrição. A MNA fornece um método simples e rápido de identificação de pacientes idosos que apresentam risco de desnutrição ou que já estão desnutridos. Identifica o risco de desnutrição antes da ocorrência de mudanças de peso ou dos níveis de proteína sérica. Ela pode ser preenchida periodicamente no ambiente comunitário e hospitalar, ou em locais de cuidados de longo prazo ${ }^{6}$.

O presente trabalho teve por objetivo a aplicação da MNA como instrumento de triagem nutricional em idosos admitidos em um hospital Dia do Idoso de Anápolis-GO com a finalidade de identificar os desafios e sua aplicabilidade na rotina da nutrição desses pacientes.

\section{METODOLOGIA}

Trata-se de um estudo de coorte prospectiva cujo tempo de acompanhamento dos pacientes foi de janeiro de 2014 a junho de 2017. A coorte pautouse em determinar a frequência de desnutrição e risco de desnutrição em grupos específicos de idosos com demências (Alzheimer, Parkinson, demência vascular ou corpos de Lewy) comparadas a idosos sem demência ou transtorno cognitivo leve. Os critérios para definição de desnutrição ou risco foram baseados no questionário Mini Nutritional Assessment (MNA) e avaliados segundo o escore: 12 a 14 pontos (normal); 12 a 11 (sob risco de desnutrição); 0 a 7 (desnutrido). A população do 
estudo foi baseada em 300 idosos com faixa etária entre 60 e 95 anos, com e sem demência, que realizavam seguimento ambulatorial contínuo em um hospital de referência para idosos em Anápolis-Goiás. A amostra foi obtida aleatoriamente por convenção, sendo constituída por 169 idosos, número auferido pelo cálculo amostral (erro amostral 5\%, nível de confiança 95\%). Os critérios de inclusão na amostra foram: faixa etária superior a 60 anos, idosos em atendimento no hospital de referência em análise. Foram excluídos idosos que não tinham cognição suficiente para realizar o seguimento. Dos 169 idosos recrutados, 19 desistiram do estudo e 27 foram excluídos. Por fim, 123 pacientes mantiveram-se até o final do estudo.

Os pacientes foram avaliados em três momentos distintos. Inicialmente foram recrutados para uma triagem, na qual foram realizados testes neuropsicológicos para a definição da presença de demências. Para isso, o miniexame do estado mental (MEEM) foi utilizado, após a exclusão de episódio depressivo, ou outros eventos que culminassem em resultados falsos positivos no exame. Em um segundo momento, por meio de avaliações geriátricas específicas, o estado de demência foi confirmado ou refutado após exame físico detalhado e testes laboratoriais para exclusão de distúrbios que falseariam o diagnóstico de demência (delirium, deficiência de vitaminas, dentre outros). Em um terceiro momento, os pacientes foram submetidos a entrevistas seriadas para verificação do fator de risco em análise e obtidos os valores de índice de massa corporal (IMC) por meio de pesagem e obtenção de altura.

A pesquisa foi devidamente aprovada pelo comitê de ética sob o número de protocolo da CAAE: 38538414.0.0000.5076.

Foi realizada análise univariada utilizando o teste do qui-quadrado para cada uma das variáveis independentes estudadas por meio do programa para software SPSS 8.0 for Windows. A medida de associação entre a presença de determinada variável e o desfecho (demência) foi estimada por meio do risco relativo estimado.

\section{RESULTADOS}

A coleta constituiu-se de $123(\mathrm{~N})$ idosos, sendo 70 $(56,9 \%)$ do sexo feminino e $53(43,1 \%)$ do sexo masculino, com faixa etária entre 60 e 95 anos. Nesse estudo, a escassa escolaridade entre os idosos obteve destaque com $\mathrm{N}=26$ $(21,1 \%)$ de analfabetos e $\mathrm{N}=85(69,1 \%)$ de portadores de ensino fundamental, contra $\mathrm{N}=11(8,9 \%)$ concluintes do ensino médio e $\mathrm{N}=1(0,8 \%)$, do ensino superior. Justaposto à investigação da escolaridade, vale enfatizar as pequenas rendas recebidas pelos pesquisados, em que $\mathrm{N}=104(84,6 \%)$ e $\mathrm{N}=17(13,8 \%)$ destes apresentavam renda de até um ou entre dois e três salários mínimos, respectivamente. Em contraponto, $\mathrm{N}=1(0,8 \%)$ e $\mathrm{N}=1$ $(0,8 \%)$ apresentavam renda de quatro ou cinco e mais de seis salários mínimos, respectivamente.

Ademais, a presença de comorbidades implicou cifras importantes. Nesse fragmento, $\mathrm{N}=34$ (27,6 \%) apresentavam Hipertensão Arterial Sistêmica (HAS), $\mathrm{N}=11(8,9 \%)$ apresentavam Dislipidemia (DL), N=3 (2,4 \%) apresentavam Diabetes Melitus (DM) e N = 41 (33,3\%) apresentavam duas ou mais associadas. Em contraste, apenas $\mathrm{N}=34(27,6 \%)$ negaram a presença dessas. Esses dados são reiterados abaixo pelo Gráfico 1: 


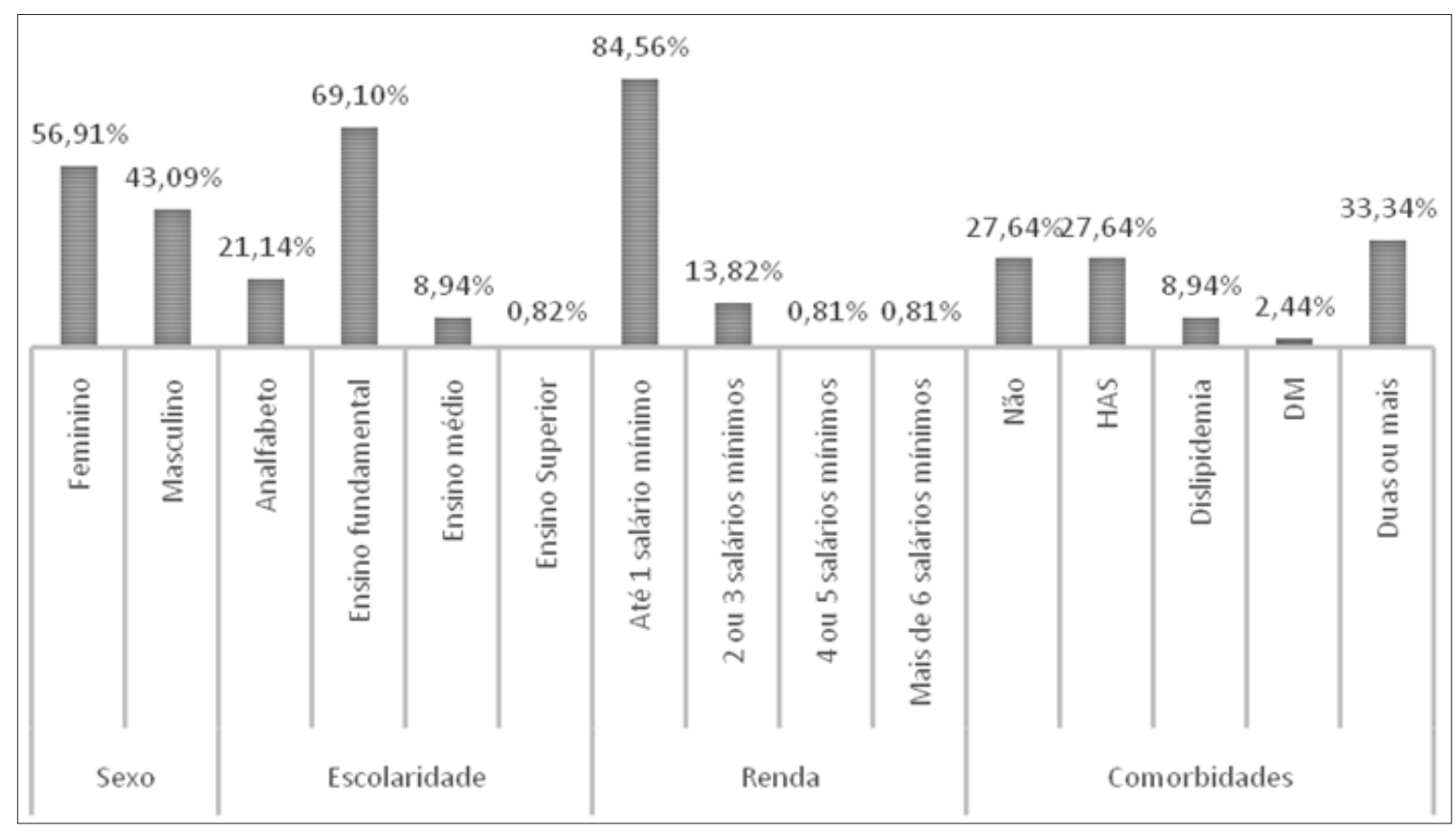

Gráfico 1. Características dos pacientes com Parkinson em uma unidade de referência em Anápolis-Goiás, 2017

Com relação à nutrição senil, observa-se preocupante risco de desnutrição ou desnutrição em pacientes com demência. No mal de Alzheimer, obtevese $\mathrm{N}=2(18,4 \%)$ dos pacientes afetados em estado de desnutrição e com risco de desnutrição $\mathrm{N}=7$ (63,2\%). Já na doença de Parkinson, os primeiros percentis anteriores representam $\mathrm{N}=3(33,3 \%)$, enquanto os segundos $\mathrm{N}=4$ $(44,4 \%)$. Por fim, as doenças vasculares também merecem atenção, posto que o risco de desnutrição foi de $\mathrm{N}=5$ (41,6\%), enquanto que a desnutrição afetou $\mathrm{N}=4$ (25\%) dos entrevistados, como pode ser observado no Gráfico 2 .

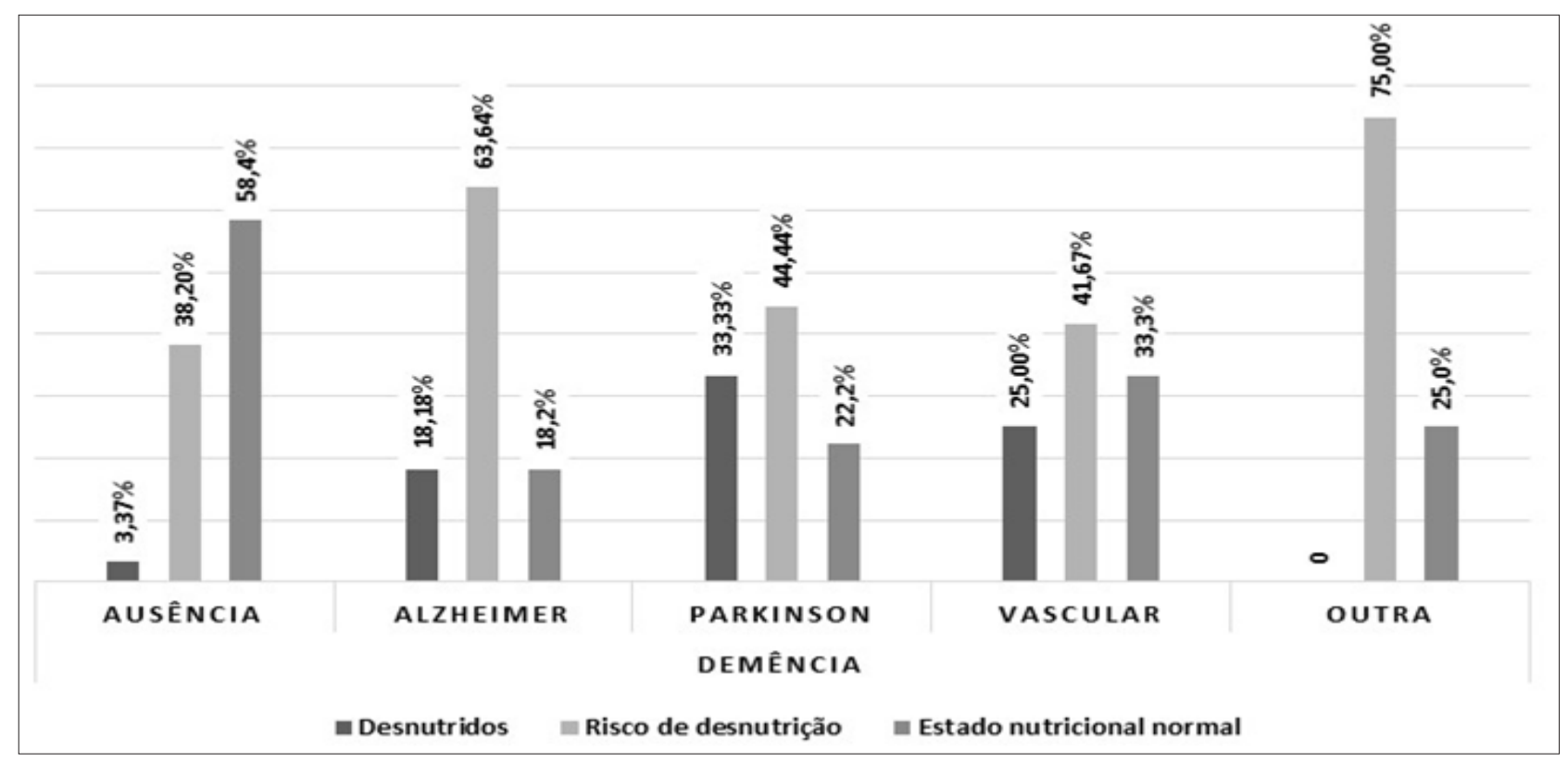

Gráfico 2. Estado nutricional de pacientes com ou ausência de demência.

*Nota: $\mathrm{p}<0.001$; teste estatístico: qui-quadrado. 


\section{DISCUSSÃO}

O estudo revelou que a demência esteve relacionada ao risco e à desnutrição em idosos institucionalizados. O estado nutricional normal foi mais prevalente em idosos sem demência $(58,4 \%)$, enquanto a prevalência entre idosos com demência foi de 18,2\% a 33,3\%. Idosos com doença de Parkinson estiveram mais relacionados à desnutrição (33,3\%) que idosos com outras demências (Alzheimer com 18,2\% e vascular com 25\%). Os pacientes com doença de Alzheimer, por sua vez, foram os que apresentaram maior risco de desnutrição (63,6\%).

Uma revisão sistemática recente buscou identificar os fatores de risco para má nutrição em idosos. A fragilidade, a polifarmácia excessiva (definida como tomar $\geq 10$ medicamentos), o declínio funcional, dificuldade de andar de escada (para pessoas $<75$ anos), declínio na capacidade cognitiva e doença de Parkinson (DP) foram correlacionados positivamente com a má nutrição?

Em concordância com os achados desta pesquisa, um estudo recente comprovou que o estado nutricional seria um fator modificável na progressão de demências. Foram avaliadas 292 pessoas com demência ( $72 \%$ de doença de Alzheimer, 56\% do sexo feminino) por seis anos utilizando o Mini-Exame do Estado Mental (MEEM), e o Mini NutritionalAssessment (MNA). O menor escore de MNA previu uma taxa de declínio mais rápida no $\operatorname{MEEM}(\mathrm{p}=0,017)^{8}$.

Um estudo realizado com 310 pacientes, dos quais 54,2\% eram mulheres, com idade média de 80,1 anos, identificou que dentre os fatores associados à presença de desnutrição moderada a grave, o sexo feminino (oddsratio: 1,7; 95\%: 1,1 a 2,8), idade superior a 80 anos (OR: 2,0, IC 95\%: 1,2 - 3,5) e demência (OR: 2,4; IC 95\%: 1,2 - 5,2) são os mais proeminentes?.

Yildiz et al. ${ }^{10}$, por sua vez, incluiram 75 pacientes com idade média de 79 anos. A maioria dos pacientes apresentava demência leve ou moderada. Esse estudo revelou que a desnutrição estaria associada ao aumento das taxas de hospitalização e quedas, disfagia, insônia, agitação, delírios, alucinações, imobilidade e incontinência. Foi independente a correlação entre MNA e o escore para demência leve.
Na Dinamarca, Sparre-Sorensen et al. ${ }^{11}$ em uma pesquisa concluíram que durante o período de janeiro de 1999 a janeiro de 2007, um aumento estatisticamente significativo no número de óbitos relacionados à desnutrição foi encontrado entre idosos com demência. Foi encontrado um total de 345 indivíduos com demência que foram a óbito pelo estado nutricional.

Uma análise transversal feita por Droogsma et al. ${ }^{12}$ avaliou 312 pacientes com demência de Alzheimer (DA), averiguando que a prevalência de desnutrição nessa população seria de $14,1 \%$. Os pacientes com DA e que apresentaram maior nível de dependência nas atividades complexas e básicas de vida diária, foram constatados como mais susceptíveis a desenvolverem desnutrição, porém esse grau de comprometimento no funcionamento de atividades diárias foi independentemente relacionado ao estado nutricional (MNA) $(p=0,001, B=-0,062)$.

Conquanto, Guerin et al. ${ }^{6}$ avaliaram 513 idosos com Alzheimer a fim de determinar os níveis de desnutrição utilizando como ferramenta principal o MNA. Destes, $79,3 \%(\mathrm{~N}=445)$ possuíam escore do $\mathrm{MNA} \geq 24$ (idosos bem nutridos), 17,8\% ( $\mathrm{N}=100)$ com escore entre 17,5 e 23,5 (risco de desnutrição), e 2,9\% (N=16) tinham escore $\leq 17$ (desnutridos).

Quanto à doença de Parkinson (DP), especificamente, um estudo revelou que a prevalência de desnutrição seria estimada em $0 \%$ a $24 \%$, enquanto que 3\% a $60 \%$ dos pacientes com DP apresentam risco de desnutrição, de acordo com o MNA. O estudo evidenciou ainda que os fatores que afetam o estado nutricional seriam a idade, sintomas motores e severidade do estágio da doença ${ }^{13}$. Os achados deste estudo corroboram com os encontrados por esta pesquisa.

\section{CONCLUSÃO}

Por fim, esta pesquisa evidenciou relação positiva entre o desenvolvimento de desnutrição e das demências, principalmente associada à doença de Parkinson. Esta doença apresenta também elevado risco de desnutrição, sendo necessários rastreamento e avaliação constantes do estado nutricional dos idosos com demência para redução da morbimortalidade nesta população. 


\section{REFERÊNCIAS}

1. Burlá C, Camarano AA, Kanso S, Fernandes D, Nunes R. Panorama prospectivo das demências no Brasil: um enfoque demográfico. Cien Saude Colet. 2013;18(10): 2949-56.

2. Chaimowicz F. Saúde do Idoso. NESCON/UFMG Curso de Especialização em Atenção Básica em Saúde da Família. Belo Horizonte: Coopmed 2009; 172:1626.

3. Bastos FC. A influência da nutrição na resposta inflamatória e no envelhecimento (Dissertação de Mestrado); 2015.

4. Najas M, Yamatto TH. Avaliação do estado nutricional de idosos. Nestlé nutrition; 2008. 7p. Disponível em: http://www. nestle-nutricaodomiciliar. com. br/Files/ documentos/AVALIACAO\% 20EST\% 20NUT. pdf.

5. Sousa APGD, Gallello DC, Lú A, Carreira MC. Triagem nutricional utilizando a Mini Avaliação Nutricional versão reduzida: aplicabilidade e desafios. Geriatr Gerontol Aging. 2015;9(2)49-53.

6. Guerin O, Soto ME, Brocker P, Robert PH, Benoit M, Vellas B. Nutritional status assessment during Alzheimer's disease: results after one year (the REAL French Study Group). J Nutr Health Aging. 2005;9(2):81-4.

7. Fávaro-Moreira NC, Krausch-Hofmann S, Matthys C, Vereecken C, Vanhauwaert E, Declercq A, Duyck J, et al. Risk Factors for Malnutrition in Older Adults: A Systematic Review of the Literature Based on Longitudinal Data. Adv Nutr. 2016;7(3):507-22.

8. Sanders C, Behrens S, Schwartz S, Wengreen H, Corcoran CD, Lyketsos CG, Tschanz JT. Nutritional status is associated with faster cognitive decline and worse functional impairment in the progression of dementia: the cache county dementia progression study 1. J Alzheimers Dis. 2016;52(1):33-42.

9. Rentero LR, Iniesta CN, Gascón JC, Tomás CJ, Sánchez CÁ. Malnutrition in the elderly patient to hospital admission, an old problem unsolved. Nutr Hosp. 2015;32(5):2169-77.

10. Yildiz D, Pekel NB, Kilic AK, Tolgay EN, Tufan F. Malnutrition is associated with dementia severity and geriatricsyndromes in patients with Alzheimer disease. Turk J Med Sci. 2015;45(5):1078-81.

11. Sparre-Sorensen M, Kristensen G. Alzheimer's disease in the Danish malnutrition period 19992007. J Alzheimers Dis. 2015; 48(4):979-85.

12. Droogsma E, Van Asselt DZB, Scholzel-Dorenbos CJM, Van Steijn JHM, Van Walderveen PE, Van der Hooft CS. Nutritional status of community-dwelling elderly with newly diagnosed Alzheimer's disease: prevalence of malnutrition and the relation of various factors to nutritional status. J Nutr Health Aging. 2013;17(7):606-10.

13. Tomic S, Pekic V, Popijac Z, Pucic T, Petek M, Kuric TG, Kramaric RP, et al. What increases the risk of malnutrition in Parkinson's disease? J Neurol Sci. 2017; 375:235-8.

Recebido em: 27/08/2017 Aceito em: 25/05/2018 